\title{
ArcheoSciences
}

Revue d'archéométrie

39 | 2015

Varia

\section{Circulation et provenance des matériaux dans les sociétés anciennes}

Adrian L. Burke

\section{(2) OpenEdition}

1 Journals

Édition électronique

URL : https://journals.openedition.org/archeosciences/4554

DOI : 10.4000/archeosciences. 4554

ISBN : 978-2-7535-4778-0

ISSN : $2104-3728$

Éditeur

Presses universitaires de Rennes

Édition imprimée

Date de publication : 31 décembre 2015

Pagination : 234

ISBN : 978-2-7535-4776-6

ISSN : $1960-1360$

\section{Référence électronique}

Adrian L. Burke, «Circulation et provenance des matériaux dans les sociétés anciennes »,

ArcheoSciences [En ligne], 39 | 2015, mis en ligne le 31 décembre 2015, consulté le 22 juillet 2021.

URL : http://journals.openedition.org/archeosciences/4554; DOI : https://doi.org/10.4000/

archeosciences. 4554 
Dillmann P., Bellot-Gurlet L. (dir.), 2014. Circulation et provenance des matériaux dans les sociétés anciennes, Paris, Éditions des archives contemporaines, coll. «Sciences Archéologiques», 344 p. (version numérique en pdf disponible).

Le livre est composé de 20 chapitres : une introduction, trois chapitres qui touchent aux matières lithiques (obsidienne et pierres de construction), cinq chapitres qui couvrent les analyses des minerais métallifères et métaux (alliages ferreux et cuivreux, or, argent, plomb et métal monnayé), trois chapitres sur les céramiques et porcelaines, un chapitre sur le verre (LA-ICP-MS), un chapitre sur les substances naturelles organiques (bitume, ambre, goudron), et finalement six chapitres qui expliquent l'application de techniques spécifiques (fluorescence $\mathrm{X}$, diffraction des rayons $\mathrm{X}$, activation neutronique, méthodes isotopiques par MC-ICP-MS, et cathodoluminescence). Ces derniers chapitres dédiés aux techniques analytiques spécifiques sont utiles, car ils présentent les techniques de pointe en archéométrie de façon succincte et accessible. Ils peuvent aussi servir comme manuel d'utilisateur, constituant ainsi un complément idéal aux autres chapitres.

Tous les chapitres traitent des aspects techniques des applications (par exemple l'échantillonnage, les défis analytiques ou l'instrumentation) et par extension de leur impact sur les questions de provenance. Cependant, les chapitres varient selon l'espace accordé à la question de la circulation des matériaux analysés et l'utilisation des données physicochimiques pour reconstituer des échanges ou autres aspects économiques dans le passé. Les exemples archéologiques proviennent largement d'Europe occidentale, ce qui reflète sans doute l'origine institutionnelle des auteurs. Il aurait été souhaitable d'avoir plus d'exemples provenant d'autres régions du monde. Les bibliographies sont aussi assez variables dans leur couverture de la matière traitée.

Les textes sont tous bien écrits, clairs et faciles à suivre pour un public de niveau universitaire. Certains sont même accessibles pour le grand public. La qualité des graphiques et des photos est généralement très bonne. Par contre, certains graphiques sont petits et il est difficile de distinguer les symboles, ce qui diminue leur utilité. Les lecteurs qui auront accès à la version numérique du livre pourront par contre agrandir ces graphiques afin de mieux les lire. Les tableaux sont nombreux et certains sont particulièrement utiles, notamment ceux qui présentent un résumé des techniques applicables par matières (par exemple l'obsidienne ou le cuivre).

Trois éléments reviennent souvent dans ce livre : l'importance de la géologie et des référentiels, le décorticage des étapes de la chaîne opératoire, et les défis analytiques reliés à chaque instrument et matériau. Ce sont des aspects de la recherche archéométrique auxquelles nous sommes tous confrontés. Ce que je trouve particulièrement rassurant, c'est la manière sobre et accomplie par laquelle ces énormes défis sont présentés par les auteurs. En fait, on sent que nous sommes rendus à une étape de maturité de la discipline qui nous permet enfin de bien saisir les limites, mais aussi l'incroyable potentiel des analyses archéométriques pour l'interprétation des sociétés du passé.

Lélément que j'ai apprécié le plus dans ce livre est la combinaison des techniques de fine pointe avec les questions d'ordre socioéconomique, telle la circulation des matériaux et objets, et leur signification et interprétation. Il est souvent difficile de trouver un équilibre en archéométrie entre ces deux aspects de la discipline. Finalement, j'enseigne l'archéométrie dans une université francophone et il m'est difficile de trouver des ouvrages de référence en français qui peuvent servir comme manuels de base pour mes cours. Cette publication est en fait un excellent livre pour un cours d'introduction à l'archéométrie des matériaux et je le recommande chaudement à tous nos collègues francophones intéressés par les archéosciences.

Adrian L. Burke Université de Montréal 